\title{
The Galaxy Evolution Explorer
}

\section{Christopher Martin, Thomas Barlow, William Barnhart, Luciana Bianchi, Brian K. Blakkolb, et al.}

Christopher Martin, Thomas Barlow, William Barnhart, Luciana Bianchi, Brian K. Blakkolb, Dominique Bruno, Joseph Bushman, Yong-Ik Byun, Michael Chiville, Timothy Conrow, Brian Cooke, Jose Donas, James L. Fanson, Karl Forster, Peter G. Friedman, Robert Grange, David Griffiths, Timothy Heckman, James Lee, Patrick N. Jelinsky, Sug-Whan Kim, Siu-Chun Lee, Young-Wook Lee, Dankai Liu, Barry F. Madore, Roger Malina, Alan Mazer, Ryan McLean, Bruno Milliard, William Mitchell, Marco Morais, Patrick F. Morrissey, Susan G. Neff, Frederic Raison, David Randall, Michael Rich, David Schiminovich, Wes Schmitigal, Amit Sen, Oswald H. W. Siegmund, Todd Small, Joseph M. Stock, Frank Surber, Alexander Szalay, Arthur H. Vaughan, Timothy Weigand, Barry Y. Welsh, Patrick Wu, Ted Wyder, C. Kevin Xu, Jennifer Zsoldas, "The Galaxy Evolution Explorer," Proc. SPIE 4854, Future EUV/UV and Visible Space Astrophysics Missions and Instrumentation, (24 February 2003); doi: 10.1117/12.460034 


\title{
The Galaxy Evolution Explorer
}

Christopher Martin ${ }^{\mathrm{a}}$, Tom Barlow ${ }^{\mathrm{a}}$, William Barnhart ${ }^{\mathrm{k}}$, Luciana Bianchi ${ }^{\mathrm{d}}$, Brian Blakkolb ${ }^{\mathrm{b}}$, Dominique Bruno $^{\mathrm{k}}$, Joe Bushman ${ }^{\mathrm{k}}$, Yong-Ik Byun ${ }^{\mathrm{h}}$, Mike Chiville $^{\mathrm{k}}$, Tim Conrow ${ }^{\mathrm{a}}$, Brian Cooke ${ }^{\mathrm{b}}$, Jose Donas $^{c}$, James Fanson ${ }^{\mathrm{b}}$, Karl Forster ${ }^{\mathrm{a}}$, Peter G. Friedman ${ }^{\mathrm{a}}$, Robert Grange ${ }^{\mathrm{c}}$, Dave Griffiths ${ }^{\mathrm{k}}$, Tim Heckman ${ }^{\mathrm{d}}$, James Jee ${ }^{\mathrm{h}}$, Patrick Jelinsky ${ }^{\mathrm{g}}$, Sugwhan Kim ${ }^{\mathrm{h}}$, Siu-Chun Lee ${ }^{\mathrm{j}}$, Young-Wook Lee ${ }^{\mathrm{h}}$, Dankai Liu $^{\mathrm{b}}$, Barry Madore ${ }^{\mathrm{f}}$, Roger Malina ${ }^{\mathrm{g}, \mathrm{c}}$, Alan Mazer ${ }^{\mathrm{b}}$, Ryan McLean ${ }^{\mathrm{a}}$, Bruno Milliard ${ }^{\mathrm{c}}$, William Mitchell ${ }^{\mathrm{k}}$, Marco Morais ${ }^{\mathrm{a}}$, Patrick Morrissey ${ }^{\mathrm{a}}$, Sue Neff ${ }^{\mathrm{i}}$, Frederic Raison ${ }^{\mathrm{a}}$, David Randall ${ }^{\mathrm{b}}$, Mike Rich $^{\mathrm{e}}$, David Schiminovich ${ }^{\mathrm{a}}$, Wesley Schmitigal ${ }^{\mathrm{b}}$, Amit Sen ${ }^{\mathrm{b}}$, Oswald Siegmund ${ }^{\mathrm{g}}$, Todd Small $^{\mathrm{a}}$, Joe Stock ${ }^{\mathrm{g}}$, Frank Surber ${ }^{\mathrm{b}}$, Alex Szalay ${ }^{\mathrm{d}}$, Arthur Vaughan ${ }^{\mathrm{b}}$, Tim Weigand ${ }^{\mathrm{k}}$, Barry Welsh ${ }^{\mathrm{g}}$, Patrick Wu ${ }^{\mathrm{b}}$, Ted Wyder ${ }^{\mathrm{a}}$, C. Kevin Xu ${ }^{\mathrm{a}}$, Jenny Zsoldas ${ }^{\mathrm{k}}$

${ }^{\mathrm{a}}$ California Institute of Technology, ${ }^{\mathrm{b}} \mathrm{JPL}$, ${ }^{\mathrm{c}}$ Laboratoire Astrophysique Marseille, ${ }^{\mathrm{d}} \mathrm{J}$ ohns Hopkins University, ${ }^{\mathrm{e}} \mathrm{UCLA},{ }^{\mathrm{f}}$ Carnegie Institute of Washington, ${ }^{\mathrm{g}} \mathrm{U}$.C.Berkeley, ${ }^{\mathrm{h}}$ Yonsei University, ${ }_{\mathrm{i}}^{\mathrm{i}} \mathrm{GSFC}$, ${ }^{\mathrm{j}}$ Applied Sciences Lab, Inc., ${ }^{\mathrm{k}}$ Orbital Sciences Corporation

\begin{abstract}
The Galaxy Evolution Explorer (GALEX), a NASA Small Explorer Mission planned for launch in Fall 2002, will perform the first Space Ultraviolet sky survey. Five imaging surveys in each of two bands (1350-1750 and 1750$2800 \AA$ ) will range from an all-sky survey (limit $\mathrm{m}_{\mathrm{AB}} \sim 20-21$ ) to an ultra-deep survey of 4 square degrees (limit $\mathrm{m}_{\mathrm{AB}} \sim 26$ ). Three spectroscopic grism surveys $(\mathrm{R}=100-300)$ will be performed with various depths $\left(\mathrm{m}_{\mathrm{AB}} \sim 20-25\right)$ and sky coverage (100 to 2 square degrees) over the $1350-2800 \AA$ band. The instrument includes a $50 \mathrm{~cm}$ modified Ritchey-Chrétien telescope, a dichroic beam splitter and astigmatism corrector, two large sealed tube microchannel plate detectors to simultaneously cover the two bands and the 1.2 degree field of view. A rotating wheel provides either imaging or grism spectroscopy with transmitting optics. We will use the measured UV properties of local galaxies, along with corollary observations, to calibrate the UV-global star formation rate relationship in galaxies. We will apply this calibration to distant galaxies discovered in the deep imaging and spectroscopic surveys to map the history of star formation in the universe over the red shift range zero to two. The GALEX mission will include an Associate Investigator program for additional observations and supporting data analysis. This will support a wide variety of investigations made possible by the first UV sky survey.
\end{abstract}

\section{SCIENCE OVERVIEW}

The primary goals of GALEX are to address these questions:

Local Universe Investigation: What are the UV properties of local galaxies, and how do rest UV properties, measured at high redshift by HST and NGST in their search for galaxy origins relate to star formation rate (SFR), extinction, metallicity, and burst history?

Star Formation History Investigation: What is the star formation and metal production history of galaxies over the redshift range $0<z<2$ ? When and where did stars and elements we see today have their origins? And does this history explain the dramatic evolution suggested in previous surveys? What global (galaxy-wide) factors drive star formation and its evolution in galaxies?

UV astrophysics: What are the statistical properties and evolutionary history of quasi-stellar objects, post-mainsequence stars, and degenerate binary stars? What is the nature of the UV rising flux in early-type galaxies? What is the rest EUV spectral energy distribution of quasars? How many quasars can be detected in their rest EUV for intergalactic medium studies by HST and FUSE? What makes up the diffuse UV background? 
GALEX addresses these goals with a set of focused objectives:

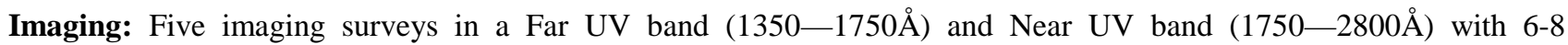
arcsecond resolution (80\% encircled energy) and 1 arcsecond astrometry, and a cosmic UV background map.

[AIS:] An All-sky Survey to 20-21 ${ }^{\mathrm{m}}$ (AB), netting $~ 10,000$ galaxies within $70 \mathrm{Mpc}$ and 10 million galaxies overall for an unbiased local calibration of UV galaxy morphology, SFR, and extinction.

[MIS:] A Medium Imaging Survey over 1000 square degrees to $23^{\mathrm{m}}$ (AB) to provide data on galaxies at intermediate distances and luminosities.

[DIS:] A Deep Imaging Survey over 80 square degrees to $25^{\mathrm{m}}$ (AB) to provide photometric redshifts, extinction and SFR for faint and distant galaxies. DIS regions will overlap SIRTF Legacy SWIRE fields.

[UDIS:] An Ultra-deep Imaging Survey over 4 square degrees to $26^{\mathrm{m}}$ (AB) to provide photometric redshifts, extinction and SFR for the faintest and most distant galaxies.

[NGS:] Nearby Galaxy Survey of 150 nearby galaxies with exposures of 1-2 orbits per galaxy.

Spectroscopy: Three overlapping slitless-grism spectroscopic surveys over the $1350-2800 \AA$ band with $\lambda / \Delta \lambda \sim 100$, resulting in greater than 100,000 galaxies with redshifts $(0<z<2)$, extinction, and SFR.

[WSS:] A Wide-field Spectroscopic Survey to $20^{\mathrm{m}}(\mathrm{AB})$ over 80 square degrees to calibrate the global UV/SFR/Extinction relations and find the rarest and most luminous star-forming galaxies. WSS will overlap DIS fields.

[MSS:] A Medium-deep Spectroscopic Survey to $21-23^{\mathrm{m}}$ (AB) over 8 square degrees to find star forming galaxies of intermediate SFR and redshift.

[DSS:] A Deep Spectroscopic Survey to $22-24^{\mathrm{m}}$ (AB) over 2 square degrees to find the galaxies with the lowest SFR and highest $\mathrm{z}$, overlapping the deepest ground-based surveys.

Associate Investigator Program: Four months of dedicated associate investigator observations will be performed in the last eight months of the nominal 28 month mission for science complementary to the primary survey goals.

Table 1 - Survey Summary

\begin{tabular}{|c|c|c|c|c|c|c|c|c|}
\hline \multirow{6}{*}{ 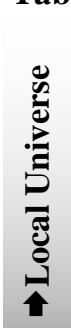 } & \multirow[t]{2}{*}{ Survey } & \multicolumn{3}{|c|}{ Survey Parameters } & \multicolumn{3}{|c|}{ Science Objective } & \multirow[b]{2}{*}{$\langle\mathbf{z}\rangle$} \\
\hline & & $\begin{array}{c}\text { Area } \\
{\left[\mathrm{deg}^{2}\right]}\end{array}$ & $\begin{array}{c}\text { Length } \\
\text { [Month] }\end{array}$ & $\begin{array}{l}\text { Expos } \\
\text { [ksec] }\end{array}$ & $\begin{array}{l}\text { Mag. Lim } \\
{\left[\mathrm{m}_{\mathrm{AB}}\right]}\end{array}$ & $\begin{array}{c}\text { \#Gals } \\
\text { (est.) }\end{array}$ & $\begin{array}{l}\text { Volume } \\
{\left[\mathrm{Gpc}^{3}\right]}\end{array}$ & \\
\hline & All-sky (AIS) & 40,000 & 4 & 0.1 & 20.5 & $10^{7}$ & 1.5 & 0.2 \\
\hline & Wide Spectroscopic (WSS) & 80 & 4 & 40 & 20 & $10^{4-5}$ & 0.03 & 0.15 \\
\hline & Nearby Galaxies (NGS) & --- & 0.5 & 2 & $\begin{array}{c}27.5 \\
{\left[\mathrm{mag} \operatorname{arcsec}^{-2}\right]}\end{array}$ & 100 & --- & -- \\
\hline & Medium Imaging (MIS) & 1000 & 2 & 400 & 23 & $3 \times 10^{6}$ & $\sim 1$ & 0.6 \\
\hline$\overline{7}$ & $\begin{array}{l}\text { Medium } \\
\text { Spectroscopic (MSS) }\end{array}$ & 8 & 2 & 400 & $\begin{array}{r}21.5[\mathrm{R}=100] \\
23.3[\mathrm{R}=20]\end{array}$ & $10^{4-5}$ & 0.03 & 0.5 \\
\hline & Deep Spectroscopic (DSS) & 2 & 4 & 2000 & $\begin{array}{c}22.5[\mathrm{R}=100] \\
24.3[\mathrm{R}=20]\end{array}$ & $10^{4-5}$ & 0.05 & 0.9 \\
\hline & Deep Imaging (DIS) & 80 & 4 & 40 & 25 & $10^{7}$ & 1.0 & 0.85 \\
\hline & $\begin{array}{l}\text { Ultra-Deep } \\
\text { Imaging (UDIS) }\end{array}$ & 4 & 1 & 200 & 26 & $3 \times 10^{5}$ & 0.05 & 0.9 \\
\hline
\end{tabular}

GALEX will produce an unprecedented, statistically powerful database of UV images and spectra of nearby and distant galaxies. Using UV properties, supplemented by data at other wavelengths, GALEX will derive global parameters for each galaxy (star formation rate, extinction, initial mass function (IMF), starburst parameters) vs. redshift. It will characterize the relationship of these parameters to galaxy properties (luminosity, type, metallicity, neighborhood, gas supply) by comparing statistical distributions of these parameters to cosmological models of the history of galaxies and QSOs ${ }^{1}$. GALEX is a high-priority Origins and SEU mission, tracing the origins of the majority of stars, metals, and many galaxies and galaxy disks, providing a framework for understanding HST and NGST rest UV from high redshift galaxies, and probing the drivers of galaxy and QSO evolution. GALEX will discover many of the UV objects HST must study in preparation for NGST. 


\section{MISSION}

GALEX is performed with a wide-field (1.2 degree) UV-optimized instrument consisting of a $50 \mathrm{~cm}$ modified RitcheyChrétien telescope, a selectable imaging window or grism, a dichroic beam splitter and corrector, a far ultraviolet and near ultraviolet sealed tube microchannel plate detectors, and support electronics. The instrument is coupled to a Orbital Sciences Corporation spacecraft that is three-axis stabilized, with fixed GaAs solar panels, a NiH battery, an X-band transmitter and S-band transmitter and receivers. The satellite mass is $277 \mathrm{~kg}$, and orbit averaged power is $279 \mathrm{~W}$. The satellite is shown during integration in Figure 1, with solar panels in the deployed position.

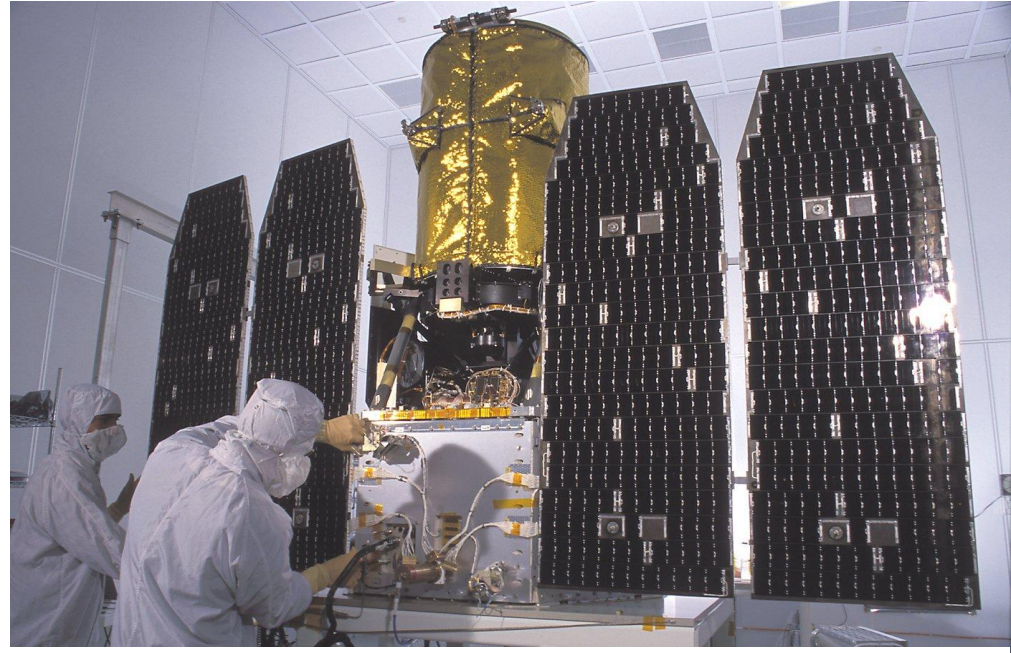

Figure 1 - GALEX satellite.

GALEX will be launched by a Pegasus-XL vehicle into a 29 degree inclination, $690 \mathrm{~km}$ circular orbit. After a one month in-orbit checkout period, GALEX will begin nominal operations. The eight surveys listed in Table 1 will be performed concurrently for the first 28 months. During the last 8 months $50 \%$ of the mission time will be devoted to an Associate Investigator Program. The mission design is simple. The standard orbital sequence for every target is shown in Figure 2. All science data is obtained only on the night side. On the day side of each 96 minute orbit, the satellite will face the solar panels toward the sun. As the satellite enters twilight, it will slew to one of the survey targets. The imaging window or grism will be selected for imaging or spectroscopic targets. If the target is spectroscopic, the grism rotation will also be selected. Once the target is reached, the detector high voltage will be ramped and the target observed.

The attitude will be dithered in a 1 arcminute spiral for deep targets, while the AIS will be obtained by scanning at 200 arcseconds/sec. Dithering and scanning is performed to average over detector non-uniformities and to prevent microchannel plate detector gain fatigue by UV bright stars. During science data collection, individual photon events are collected by the far ultraviolet and near ultraviolet detectors and front-end electronics (FEE), formatted by the instrument Digital Processing Unit (DPU), and stored on the spacecraft solid-state tape recorder (SSR) along with housekeeping data. At the end of orbital night, detector high voltages are ramped to idle levels to protect them from damage and the spacecraft returns to solar array pointed attitude. Up to four times per 24 hour day the SSR is dumped via the X-band transmitter to ground stations in Hawaii or Perth, Australia, operated by Universal Space Networks (USN). Real-time satellite health and safety monitoring is performed by the Mission Operations Center (MOC) at Orbital Sciences Corporation in Dulles, Virginia, during the ground pass. Science telemetry is shipped by ground network to the Science Operations Center at Caltech, with a latency of 4 hours for housekeeping and 48 hours for photon data. Science data will be processed at Caltech to produce images, object catalogs, and extracted spectra. Catalogs and spectra will be delivered to the Space Telescope Science Institute to be archived in a database developed by Johns Hopkins University for the Sloan Digital Sky Survey (SDSS). 


\section{INSTRUMENT}

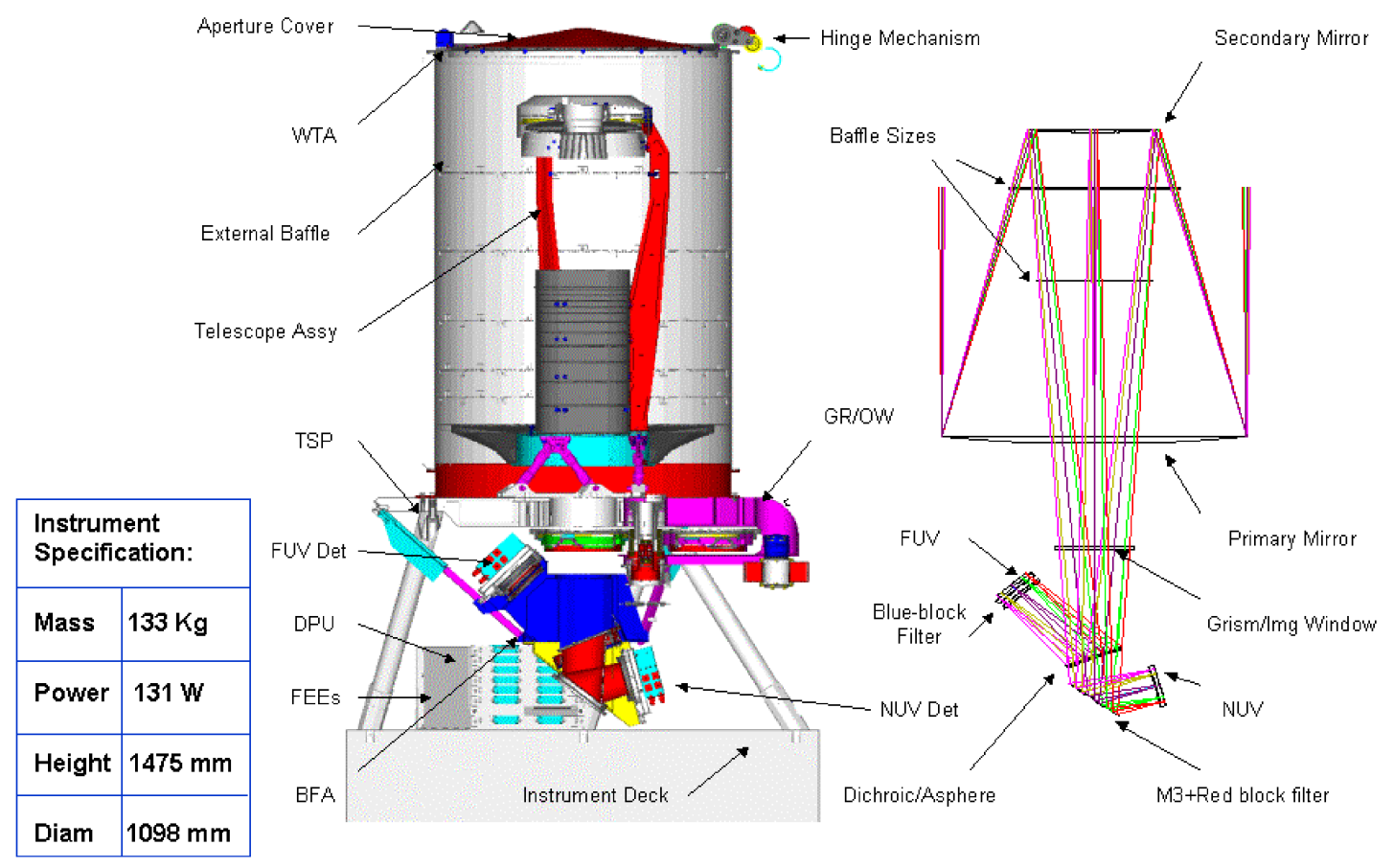

Figure 3 - GALEX instrument and optical design.

\subsection{Optical Design}

The adopted design meets the challenging requirements of providing $2-3 \operatorname{arcsec} 80 \%$ encircled energy (80\% EE) optics resolution with a high throughput, large field of view $(65 \mathrm{~mm}$ or $1.24 \mathrm{deg}$ ) in four different optical paths (two simultaneous UV channels, with selectable imaging and slitless spectroscopy modes), while keeping the instrument compact, simple to build and adjust. The pupil diameter is essentially limited to half a meter by the available volume in the Pegasus faring. As needed for a SMEX program, special attention has been paid in the design concept to maintaining tolerance to component positioning and orientation, especially for the moveable parts. The design minimizes the number of optical components and surfaces by employing components to serve dual purposes as correctors in the case of the grism, optical window, dichroic beam splitter and both detector entrance windows.

The concept, shown in Figure 3, is a slightly modified Ritchey-Chrétien telescope in which astigmatism is corrected by a low power fused silica aspheric window in the converging beam. This aspheric window bears a multilayer dichroic coating to separate the FUV (reflected) and NUV (transmitted) light. Its reflecting entrance side corrects the FUV channel, whereas the exit side cancels the entrance side effects and brings in the required amount of correction for the NUV channel. A small wedge on this aspheric window compensates for the coma it induces in the NUV convergent beam. $\mathrm{A} \mathrm{CaF}_{2}$ grism ahead of the aspheric corrector provides slitless spectroscopy over the whole GALEX FOV. Its wedge angle is adjusted to correct for the coma it induces in the converging beam ${ }^{2}$, simultaneously for orders 2 and 1 spectra of the FUV and NUV channels respectively. It turns out that the resulting deviation is low, a primary advantage for switching between imaging and slitless spectroscopy. This switching is performed by exchanging the grism with a low power plano-convex $\mathrm{CaF}_{2}$ imaging window. These two components are mounted on a rotatable wheel that also provides an opaque position. The grism and the imaging window positional tolerances are loose $(0.5 \mathrm{~mm})$ because they are dioptric components with only a low power. This low power is optimized to correct for the axial chromatism of all 
the transmissive elements. The field curvature is cancelled by the power in the detector windows, and the detectors are tilted to the best plane. A remarkable feature is that a single blaze angle on the grism facets is found to provide a well centered efficiency for both NUV and FUV channels, owing to the $\mathrm{CaF}_{2}$ index variation with wavelength.

The design yields a field-averaged spot size of $1.6 \operatorname{arcsec}(80 \% \mathrm{EE})$ for the FUV imagery and $2.5 \operatorname{arcsec}(80 \% \mathrm{EE})$ for the FUV spectroscopy at $1600 \AA$. NUV performance is similar. There is no in-flight refocus capability.

\subsection{Telescope}

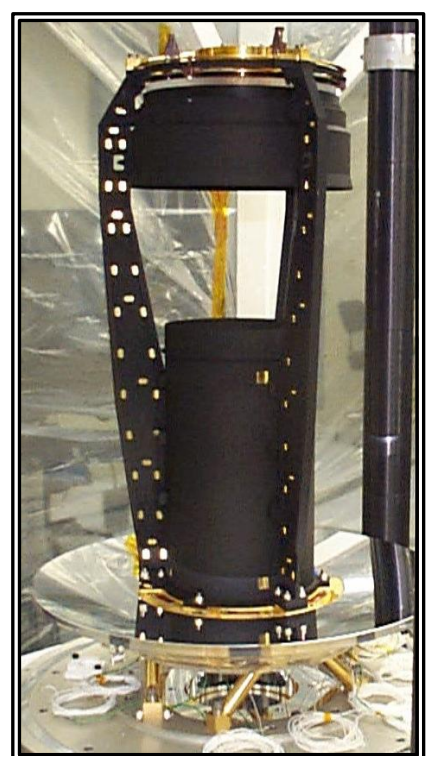

Figure 4 - GALEX

Telscope.

The telescope, shown in Figure 4, is a $50 \mathrm{~cm}$, f/6, modified Ritchey-Chrétien design optimized for the required 1.2 degree wide field of view. The telescope and flight spare (used as abeam collimator for optical testing) were originally designed and provided by Light Works Optical Corporation (LWO). The design requirements were comparatively modest, driven by overall mass, stiffness, and maintenance of the required 30 micron $80 \%$ EE spot size over flight operational temperatures with no active thermal control. The LWO design incorporates a Meinel strut arrangement with hub-mounted primary and secondary optics. Technical problems with the as-built LWO telescope required that JPL perform a complete redesign and retrofit of the primary mirror mount. The $f / 2$ primary and the secondary were ground and polished on Fused Silica blanks by Brunasche, and overcoated with $\mathrm{Al}$ and $336 \AA \mathrm{MgF}_{2}$ by the Goddard Space Flight Center Coating Laboratory. The secondary is bonded with RTV 566 to a super-invar hub, which is turn mounted to an Invar 36 spider. The spider is attached to an Invar 36 tower and primary baffle assembly. The JPL-provided primary mirror mount is an Invar 36 flexured hexapod bonded to the primary with Epoxy 2216. The mount is quasikinematic and induces no more than 0.2 waves to the system performance over flight temperatures. The flight telescope was assembled and aligned at JPL, and was subjected to vibration and thermal vacuum testing beyond expected environmental conditions. A small amount of focus shift is present over temperature, roughly 5 microns/degree $\mathrm{C}$, and due mostly to the secondary mirror mount. The secondary and primary mirrors are temperature controlled. A small amount of astigmatism (approximately 1 wave, and induced by the secondary mount) was discovered during end-to-end optical testing, but has remained stable. In spite of these deficiencies, the telescope contribution to the system angular resolution budget remains within specification.

\subsection{Window and Grism}

The dispersive component for the spectroscopy mode is a 75 groves $/ \mathrm{mm} \mathrm{CaF}_{2}$ grism that can be inserted with loose tolerances into the convergent beam to produce slitless spectra. Both the grism and the imaging window that comes in place of the grism have been polished by STIGMA from a $180 \mathrm{~mm}$ diameter, high quality UV-grade monocrystalline $\mathrm{CaF}_{2}$ ingot screened for low phosphorescence, provided by SOREM. Both the grism and imaging window have a low curvature entrance face and require a tight control of the differential thickness $( \pm 30 \mu \mathrm{m})$ to keep in focus while switching between imaging and spectroscopy. Nevertheless the most difficult part was by far the grism ruling: although grisms are widely used in visible and near infrared ground-based astronomy, the UV cutoff of the resins involved in their manufacturing process prevents their use in the vacuum UV range. LAS and Jobin-Yvon developed a proprietary process to imprint the blazed profile into the $\mathrm{CaF}_{2}$ crystal. Figure 5 shows the grism, the efficiency ratio of the flight grism to that of the imaging window, and a grism image. The absolute efficiency measurements reach $82 \%$ in the NUV and $61 \%$ in the FUV. Electromagnetic computations have shown the polarization is negligible. The GALEX grism was the first one ever built for the FUV $^{3}$. The grism is mounted in a mechanism that can rotate the spectra to 872 position angles on the detectors and sky.

Slitless spectroscopy produces numerous overlapping object spectra and multiple orders. For this reason, all spectroscopic surveys are obtained using a different position angle for each orbit integration. The confusion problem becomes worse as the surveys deepen. At the same time the number of position angles increases, compensating. The 
deep spectroscopic survey will utilize all 872 distinct position angles. Simulations have shown that this method yields a confusion limit comparable to that of the imaging surveys when more than 100 angles are utilized. Satellite roll, which may also be used to control spectral position angle, is often otherwise constrained.
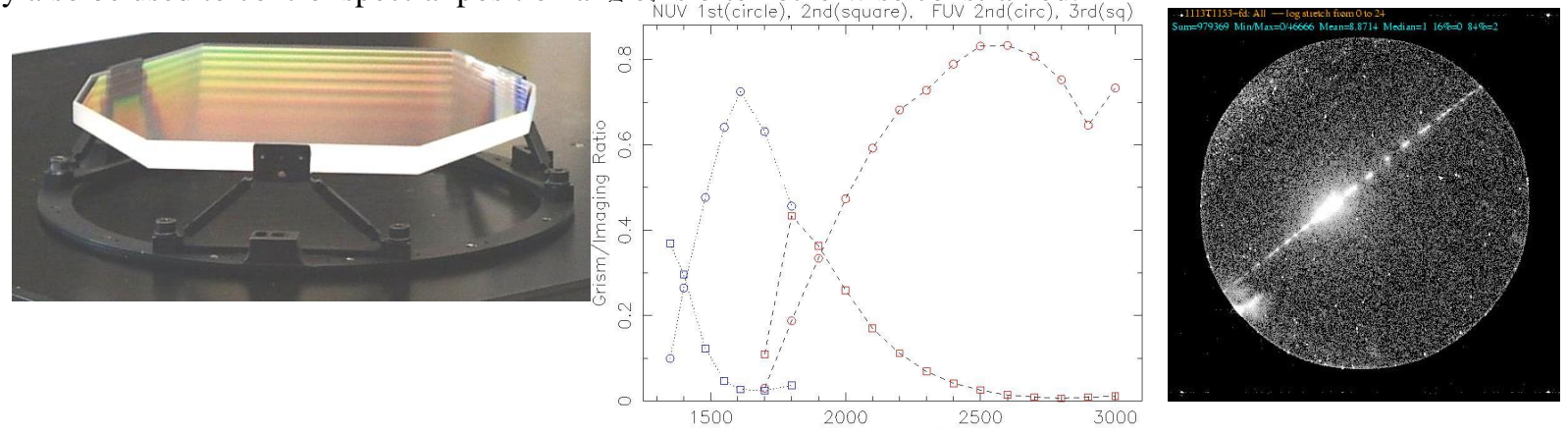

Figure 5 - LEFT: Grism. CENTER: Ratio of Grism to Imaging Window efficiency. RIGHT: Grism calibration image.

\subsection{Dichroic Beam Splitter}

The $110 \mathrm{~mm}$ diameter UV-grade fused silica aspheric corrector window (AS) is only $4 \mathrm{~mm}$ thick. Using its entrance face as a mirror sets tight manufacturing tolerances that already were challenging for polishing it flat on both faces, at the first manufacturing step. The AS is tilted at $22^{\circ}$ to the beam to accommodate the FUV detector position, but the aspherization has been performed axisymmetrically to simplify manufacturing, with only marginal impact on the image quality. The required aspherization was $4.72 \mu \mathrm{m}$ for the first side and $11.4 \mu \mathrm{m}$ for the second. The two aspherisations were achieved at IOTA (University of Paris) by broad-beam ion milling with a carbon mask, a technique that combines a fairly good shape precision, conservation of the original smoothness comparable to that allowed by the classical flat polishing, and absence of any stress in the material thus preserving the shape of the side not being processed ${ }^{4}$. The FUV side has been eroded first, on the basis of a mask iteratively adjusted from calibration erosion on thick blanks. A significant part of the FUV residual departure from planarity after polishing has been corrected for by adjusting the erosion time. For the NUV side, the erosion has been performed in two steps, the second ( $10 \%$ of the exposure time) being adjusted to optimally correct for the combined residuals of the FUV side and first NUV polishing. The overall residuals have been measured to $27 \mathrm{~nm}$ and $31 \mathrm{~nm}$ respectively on the FUV and NUV sides, bringing a negligible contribution to the global image budget.

The mechanical mount developed for the aspheric corrector window was used to hold the crystalline grism and imaging window with minimal strain in the thermal, vibration and shocks environment of Pegasus launchers. The three components were bonded to the heads of three titanium flexure bipods. The bipod leg angle was set to equally share the maximal stress in the thinned parts for the $\mathrm{X}, \mathrm{Y}$ and $\mathrm{Z}$ directions of vibration. An o-ring linking the bipod bases to a suitably flexible telescope interface has proved very efficient in damping the sharp natural resonance during environmental testing.

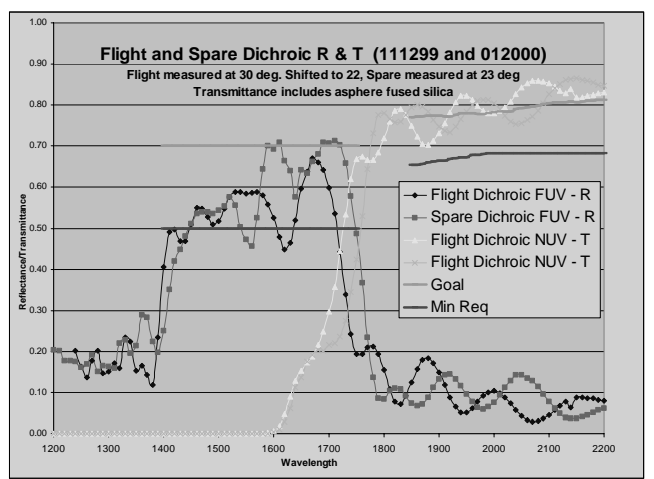

Figure 6 - Flight and spare Dichroic beam splitter reflectance and transmission.

\subsection{Filters}

Three of the GALEX back focal assembly optics are coated with multilayer filters designed to enhance the in-band throughput and offband rejection of the GALEX instrument. The dichroic coating applied to the entrance face of the fused-silica aspheric corrector plate separates the FUV (reflection) and NUV (transmission) optical paths. This alldielectric dichroic coating provides a significant improvement over conventional $40 \%-40 \%$ UV beam splitter coatings, with a mean reflectance of $61 \%$ over the $1400-1700 \AA$ band and a mean transmittance of $83 \%$ over the $1800-2750 \AA$ band. A transmissive blue-edge filter coated on $\mathrm{MgF}_{2}$ provides $10 \%$ rejection of the OI 1304 airglow line for the FUV channel. A reflective broad-band red-blocking filter on the M3 
folding mirror has an edge at $2800 \AA$. This edge yields an additional factor of 10-20 rejection for the NUV zodiacal light background above and beyond the natural $\mathrm{Cs}_{2} \mathrm{Te}$ detector photocathode cut-off.

\subsection{Detectors and Front-End Electronics}

The GALEX detector system ${ }^{5}$ is composed of two largeformat, microchannel plate amplified, sealed tube detectors with delay line readouts and associated electronics. The detectors have $65 \mathrm{~mm}$ diameter active areas and will be the largest of their kind on orbit. They were fabricated at UC Berkeley by the Experimental Astrophysics Group in collaboration with members of the Caltech Space Astrophysics Laboratory. The FUV channel has a CsI photocathode deposited directly on the $\mathrm{MCP}$ and a $\mathrm{MgF}_{2}$ window for UV transmission down to the instrument cutoff at approximately $1350 \AA$. A voltage applied to wires deposited on the window surface enhances the quantum efficiency (QE) of the detector by approximately 50\%. The NUV channel has a $\mathrm{Cs}_{2} \mathrm{Te}$ photocathode deposited on the surface of its fused silica window, which is proximity-focused on the MCP. The $\mathrm{Cs}_{2} \mathrm{Te}$ photocathode requires a sealed tube to protect it. We made the strategic decision to seal the FUV detector as well for operational simplicity during integration and

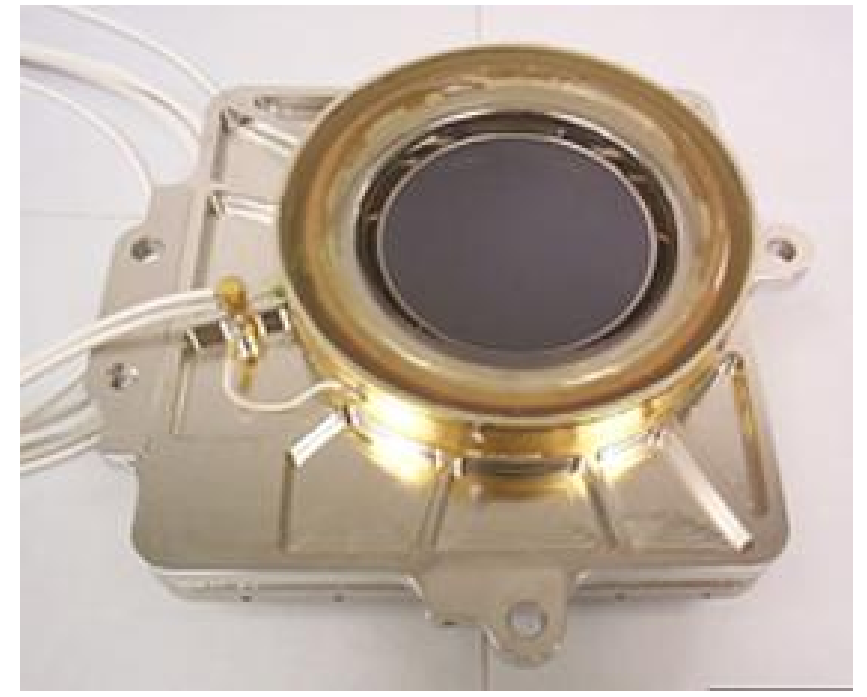

Figure 7 -- Flight NUV Detector test. In spite of the significant associated costs, the strategy has proven to be tremendously worthwhile because no ground or flight worthy vacuum pumping was required and because we could operate the detectors at any time.

The electronics for each detector include high and low voltage power supplies adapted from the SOHO program, an 8051-based controller, four high speed timing amplifiers, and an FPGA-based signal processing board and dedicated controller. Commands, housekeeping and science data are communicated between the detector and instrument via RS422 interfaces. Each detector has its own entirely independent set of electronics, with total power consumption by the dual system being approximately $75 \mathrm{~W}$. The electronics were developed by a large collaboration including Battel Engineering (power supplies), Southwest Research Institute (digitizer) and UCB (controller and amplifiers), with systems engineering and testing performed jointly by Alias Aerospace, Baja Technology and Caltech.

As of this writing the tubes have each accumulated approximately 1000 operating hours with no sign of performance degradation. They were life-tested at Berkeley prior to delivery to JPL/Caltech, undergoing approximately a week each of high count rate burn-in that was intended to be representative of approximately $10 \%$ of the mission-integrated dose estimate. These tests and our subsequent experience indicate the detectors should be relatively immune to the permanent pulse height reduction commonly observed in other microchannel plate based systems; we attribute this to the extensive UV scrubbing process required prior to sealing the getter-pumped tubes, which is of order $0.1-0.2 \mathrm{C} / \mathrm{cm}^{2}$. The system has been vibrated and thermally cycled numerous times during component, instrument, and satellite tests, including an extensive calibration program described in section 2.12. Similarly, the electronics have undergone extensive testing (and some modification) at JPL, having accumulated approximately 500 failure-free hours with the flight software version and current flight configuration. There is a single set of spare electronics and two spare detectors (one for each channel) and that are monitored periodically for functionality and background stability at JPL as part of the instrument test bed. This test bed will become one of the primary means of debugging problems and new command scripts after GALEX is on orbit.

Detector resolution tests could only be performed during end-to-end optical tests, because of the presence of the thick, curved entrance windows. The detector resolution varies significantly with position and pulse amplitude. Based on full aperture tests corrected for optical contributions to the point spread function, and sub aperture tests in which the optical contributions can be ignored, the median resolution is 75 microns for the FUV and 107 microns for the NUV detectors 
( $80 \%$ encircled energy diameter), which corresponds to 50 microns and 70 microns FWHM, respectively. NUV resolution is somewhat wavelength dependent because of photoelectron gap spread transiting the proximity-focused gap. Post-tube processing detector QE is $10 \%$ at $1500 \AA$ (FUV) and $8 \%$ at $2300 \AA$ (NUV).

\subsection{Mechanisms and Mechanical Design}

\section{Mechanical Hardware}

The GALEX Instrument's mechanical hardware consists of a Telescope Support Plate (TSP), Back Focal Assembly (BFA), Barrel Baffle and bipod supports (see figure 3). Each assembly was designed for ease of fabrication and drew on past hardware designs as a starting point, thus reducing the number of design iterations. For example the optical bench and bipod supports derived from JPL flight hardware designs from projects such as EOS-MLS and Cassini. The heritage design concept formed a good starting point, however, the final designs were modified for GALEX requirements.

The TSP is the central node of the Instrument to which all components are attached (i.e., Baffle, Grism Rotator Optical Wheel [GROW] mechanism, BFA and the bipod support). Because the instrument's alignment requirements need to be maintained throughout orbital temperature variations, the TSP plays an integral part of the athermalization design of the Telescope Assembly and the BFA. Since the optical design is radially symmetric the compensation for thermal effects is along the boresight of the optics. The BFA design is athermalized using aluminum for the bench and invar for the metering structure. The design maintains telescope alignment in the presence of either a uniform temperature change or a uniform gradient. As the aluminum BFA bench changes temperature, the optical focal point moves along the boresight. This movement is compensated for by dimensional changes in the TSP and angular adjustments in the bipods. As the temperature decreases, the TSP shrinks, moving the invar bipods inward radially thereby creating a new angle between the bipods and the bench. This moves the BFA and thus the optical focal plane back to its original position. Within the BFA, the relative position of each optical element is maintained with the same sort of athermalization design in their local mounting scheme. Each optical element is mounted on titanium bipod flexures. The aluminum bench thermal contraction changes the angle in the bipod creating a piston motion compensating for the thermally induced motion.

The baffle assembly design was designed around stray light, contamination and cover support, although the stray light was the driving design requirement. Using stray light analysis to prevent light from hitting the detector, knife-edge vanes were placed along the four-foot length of barrel and a "stop" was placed at the surface perimeter of the primary mirror. The interior surface finish of the baffle (as well as all other optical cavities) was coated with Chemglaze Z306 to meet reflective/absorbing requirements necessary for a UV instrument.

The final component of the mechanical hardware was the bipod support structure. Since the bipods supported the entire instrument and were not critical for optical alignment the high random vibration environment imposed by a Pegasus launch vehicle drove their design. Normally the bipods would be very stiff to keep the Instrument first fundamental frequency higher than the spacecraft bus structure eliminating any coupling of the two structures. Inherently, an optical system has very stiff joints to maintain the optical alignment and keep the optical interfaces from slipping. The stiff joints in the optical system unfortunately cause very high amplification throughout the telescope, resulting in high accelerations at the location of the secondary mirror assembly. Typically structural analysis and dynamic qualification programs assume the payload is a fixed base system. While this is not exactly true with the actual launch vehicle, the payload (or instrument) must still pass the dynamic environment qualification. In order to test the instrument without damaging hardware during random vibration tests the bipods were designed with a passive constrained layer viscoelastic material, which introduced damping into the relatively stiff telescope system. To further limit the test input to the low mass, highly amplified secondary mirror assembly, the frequency of the Instrument was tuned on the bipods so that the secondary mirror's primary modes aligned with the spacecraft's major modes. This achieved the desired result of reduced response in the secondary mirror primarily because the force limiting used during the test was more effective given the larger effective mass at each mode. 


\section{Mechanisms}

The GALEX Instrument has two primary mechanism assemblies, the Cover Assembly and the Grism Rotator Optical Wheel (GR/OW). The Cover assembly contained four distinct devices and the GR/OW contained two separate mechanisms. The goal was to utilize heritage designs to keep qualification costs to a minimum. Another important feature was modularity. Since mass was not a driving factor, components were designed for ease of assembly and disassembly from the system. This allowed assemblies to be bolted into the Instrument without having them be an integral part of the adjacent unit.

The Cover Assembly was a one time deployable device, which could not be jettisoned because of orbital debris considerations. The function of the Cover Assembly was to prevent contamination of the optical cavity during I\&T and launch. Other requirements included not to be a source of stray light and to have a fail safe design to not block the aperture. The design also included a vent path for Nitrogen gas purge and venting during launch ascent. The four mechanisms associated with the cover are the hook, latch, hinge, and energy-absorbing stop.

The hook and latch mechanisms were chosen because they had flight heritage from both the DS1 MICAS and MISR instruments. They incorporate a simple spring-loaded hook, which is held by a pin. The pin is part of the latch assembly of which the main component is a Wax Thermal Actuator (WTA). The WTA is a redundant, off-the-shelf, fully qualified device with space heritage. The hinge mechanism is a new fully redundant, elegant but simple design. The hinge uses a spring that pushes a nut along a Teflon-coated a helical screw drive. The screw drive rotates the cover through 270 degrees. There are two screw drives and two hinges, both using redundant bearings so the seizing of one half of the hinge will not stop the other half from rotating. Once the cover rotates through its full motion it makes contact with two identical energy absorbing mechanisms, which prevent damage to the telescope and are resetable after each use. The energy absorber is a spring compression mechanism with a locking, ratcheting device to hold the spring in place when compressed. To prevent any chattering of the deployed cover, magnets are used to hold the cover to the absorber head.

The GR/OW consists of a mechanism within a mechanism: the grism rotator sits on the optical wheel that swaps between imaging and grism modes. The functional requirements were to accurately move a larger wheel between the two optical elements and an opaque position, which were placed 120 degrees apart. When it is in the beam, the grism has to rotate 360 degrees in $~ 1 / 1000$ increments. The entire mechanism was made as a unit with its own structure so it could be installed and removed from the instrument without disrupting other optical alignments. The motor used for the Optical wheel rotation was a mini-dual drive assembly (MDDA) which contains a fully redundant brushless DC motor with flight heritage. The motor drives the wheel into hard stops at the end of travel in each direction to provide the necessary repeatability and accuracy without having to use sensors. The grism rotator mechanism uses a stepper motor, encoder and twist capsule all previously flight qualified.

\subsection{Thermal design}

A thermal control design has been successfully developed for GALEX that complies with the thermal requirements imposed on the electronic equipment and optical elements for operating and non-operating conditions. The thermal design is based on a consideration of extreme, but realistic environmental parameters and spacecraft orientations that are consistent with flight system attitude constraints. Thermal control is achieved by utilizing heaters (operational and/or replacement) on optical components and electronic equipment, radiators on electronic equipment, surface coatings, and multi-layer coating (MLI). The GALEX instrument is maintained within the non-operating temperature limits in the survival mode utilizing thermostatically controlled replacement heaters at appropriate locations. Numerous thermal tests at subsystem and system levels have validated the thermal design.

\subsection{Contamination Control}

Cost and schedule constraints of the GALEX mission did not allow for a comprehensive contamination source and transport analysis to be performed. Instead, cleanliness requirements were established through similarity by leveraging the rigorous contamination control program implemented on the second Wide-Field Planetary Camera (WFPC-II) for the 
Hubble Space Telescope mission-also an instrument with a UV component. At the system level, a ten-percent degradation in UV response was allocated to contamination: nine-percent due to molecular and one-percent due to particulate contamination. End of mission cleanliness goals were established at one-percent fractional area coverage for particulate contamination, and sixty-five $\mathrm{ng} / \mathrm{cm}^{2}$ summed over the optical train for molecular film contamination.

To achieve the stringent cleanliness requirements, we took many steps planned before and during the instrument design phase. All GALEX materials and processes were submitted to the Contamination Control Engineer for acceptance or redesign. The telescope optics are, by design, isolated from the rest of the satellite and the other components of the aft focal assembly (e.g., grism wheel stepper motor, mini dual drive, and structure) by a so called "contamination enclosure". The venting for the spacecraft bus and its electronics is in the aft direction (away from the telescope) and the spacecraft bus vent incorporate molecular adsorbers. The forward edge and two lateral edges of each solar panel are closed-out in order to reduce panel outgassing toward the instrument aperture. All the exterior MLI is vented away from the instrument aperture, the solar panels and other satellite exterior surfaces. The back-focal assembly is continuously purged, except during vacuum operations, with dry grade $\mathrm{B} \mathrm{GN}_{2}$. The primary and secondary mirrors are equipped with decontamination heaters for use in flight.

Assembly of the instrument took place in Class 10,000 and Class 1000 environments. Satellite-level integration was carried out in Class 10,000 and 100,000 environments. The hardware was bagged and purged when required to be exposed to less controlled environments. A program of optical witness mirror monitoring was implemented to assess the exposure of the instrument to environmental contaminants. The witness mirrors were coated with the same coatings as the telescope primary and secondary mirrors and reflectance was measured over the range 1216 to $3000 \AA$. A Contamination Control Working Group comprised of instrument, spacecraft, launch vehicle, launch site, and customer representatives was invaluable in coordinating the implementation of a stringent, mission-level, contamination control program on a tight project budget and schedule.

\subsection{Digital Processing Unit}

The GALEX digital processing unit (DPU) is a VME bus computer system containing a RAD6000 processor (of Pathfinder heritage) and three custom peripheral boards. The RAD6000 is a 32-bit 20 MIPS processor with 128 Mbytes of RAM and 3 Mbytes of EEPROM. A digital control board implements six RS-422 clock/data/enable serial interfaces to the NUV and FUV front end electronics (FEE), and one RS-422 16-bit parallel interface to the solid state recorder (SSR) in the spacecraft (S/C). The digital board also receives a $2 \mathrm{KHz}$ and a $1 \mathrm{~Hz}$ clock from the S/C. An analog control board contains a standard MIL-1553B command/telemetry interface to the S/C, and a custom 16-channel housekeeping (HK) hybrid that measures 13 instrument temperatures and 3 VME bus voltages.

A power control board provides regulated and filtered $+5 \mathrm{~V},+12 \mathrm{~V}$, and $-12 \mathrm{~V}$ for the $\mathrm{VME}$ bus system. It contains drivers for the Optical Wheel drive motor (MDDA), the Grism drive stepper motor, and the WTA (wax thermal actuator) used to deploy the telescope cover. It receives digital feedback from these 3 mechanisms via microswitches and halleffect sensors, and digital Grism position information from a position encoder in the Grism assembly. The power board also drives 6 operational heaters.

The DPU flight software is involved in all aspects of instrument control. Science data is received from the FEE, processed, and transmitted to the SSR. MIL-1553B commands and telemetry are continually exchanged, using an architecture employing 200 5-ms slots per second, derived from the S/C clocks. Switch and HK telemetry data are continually collected. Six of the HK channels are used for programmable heater control. Finally, the DPU controls mechanism operation via explicit commands from the CMD/TLM interface.

\subsection{Instrument Integration and Test}

The GALEX project followed a well-proven, standard JPL process in the implementation of its Integration and Testing Program. This standard process consists of component integration under quality assurance supervision and a rigorous environmental testing campaign including simulated launch vehicle vibration survivability testing, space environment 
thermal vacuum testing, and electromagnetic emission and susceptibility testing. For GALEX, this process was conducted at the component, Instrument, and Satellite level.

At the component level, microchannel plate detector subsystem components were assembled and tested for performance, stability, launch survivability and performance degradation due to electromagnetic fields. The telescope optical subsystem was tested for launch survivability and stability over temperature. In these early tests, many issues regarding design and performance were discovered and addressed. For example, measurements of performance over temperature prompted a redesign of the primary mirror mount in order to decrease primary mirror deformation over temperature to very low levels and the detector subsystem performance was found to be susceptible to relatively low field strength emissions at frequencies from 250 to $400 \mathrm{MHz}$. Several steps were taken to reduce the effect to acceptable levels.

Once tested and accepted, the components were assembled into the GALEX Instrument. Again, several space and launch environment simulation tests were conducted. With an integrated instrument, these tests evaluated system-wide functionality and indicated the performance that could be expected from GALEX on-orbit. These tests did uncover several shortfalls that were not obvious at the component level. For example, interactions in the assembled instrument structure lowered the first fundamental resonance frequency below $40 \mathrm{~Hz}$. The launch vehicle has a known, large magnitude excitation peak around $35 \mathrm{~Hz}$. In order to prevent constructive coupling that would result in large, damaging accelerations at the telescope secondary mirror, the Instrument's main bipod support structure was redesigned to raise the first mode to $47 \mathrm{~Hz}$ and damp remaining accelerations down to levels safe for the telescope assembly.

Finally at the Satellite level, the full suite of simulation testing was repeated. By this time, the GALEX spacecraft was well understood and the test proceeded without any major discoveries. In addition to the vibration, thermal vacuum and EMI/EMC testing, two additional tests, launch vehicle separation and acoustic testing were performed at this level. The launch vehicle separation test involved attaching the separation ring to the GALEX spacecraft, arming it with a live explosive bolt cutter and firing it to separate the spacecraft and launch vehicle attachment rings. Vibration levels were measured and found to be significant but safe. Acoustic testing involved surrounding the Satellite with about 30 rock concert sized speakers and blasting the Satellite with a $120 \mathrm{~dB}$ white noise acoustic spectra. Spectacular, but pretty benign for the spacecraft.

\subsection{Instrument Calibration and Performance}

From April 20 through May 13, 2002 the GALEX JPL instrument and Caltech science teams performed a thermalvacuum ground calibration of the GALEX instrument. We operated around the clock in three overlapping, equivalent shifts per day. We made measurements continuously at cold, nominal, and hot operational thermal plateaus, and during transitions between them. We successfully obtained required data and did not have any failures that

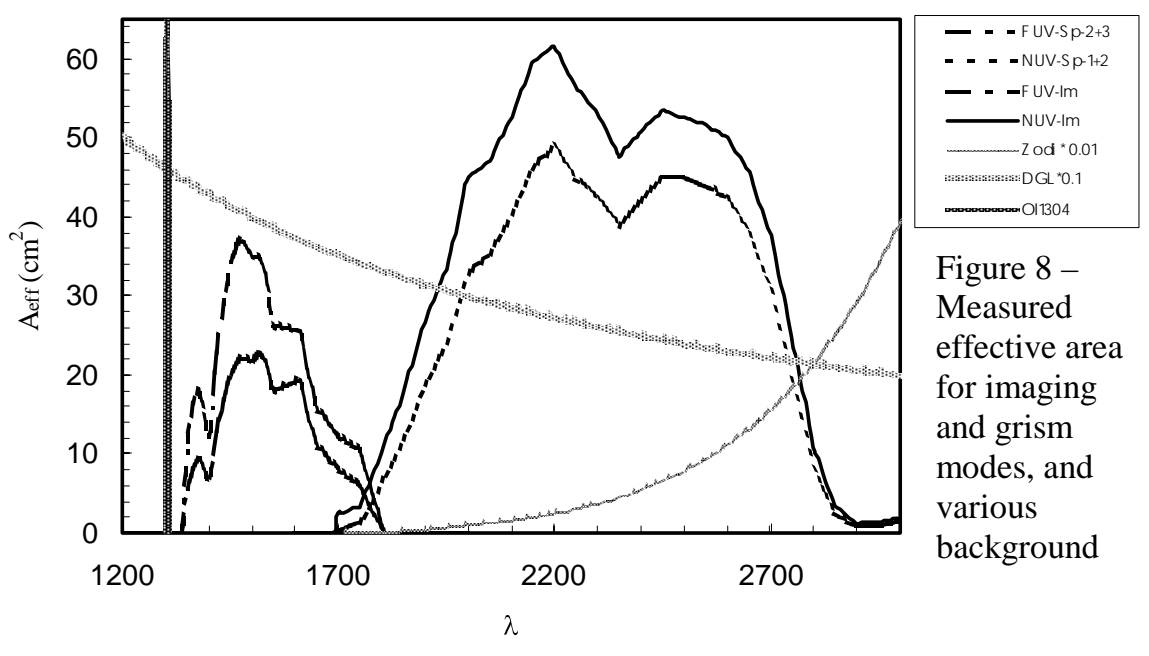
required us to break vacuum. We collected 100 GByte of data during $~ 500 \mathrm{hr}$ when at least one detector was at high voltage. We encountered no surprises and there was no damage to the instrument.

The primary purpose of the calibration thermal vacuum test was to make detailed measurements of GALEX instrument performance characteristics. The flight data-analysis pipeline requires these as inputs. The second purpose was to look for anomalies while thoroughly exercising the instrument over a range of targets, light levels, count rates, temperatures, 
bus voltage, and detector high voltage. The third purpose was to verify performance of parameters including proper focus and optical alignment, point spread function (PSF), detector spatial nonlinearity, and thermal stability, as required by our requirements matrix. The fourth purpose was to test a modification of a survival heater thermostat and to perform two thermal vacuum cycles (of four total) at the instrument level to demonstrate thermal robustness and to look for workmanship problems. The fifth purpose was to exercise the science operations and data analysis team, the instrument team, and the data analysis tools. The sixth purpose was to select table values for operating the detector front end electronics (FEE).

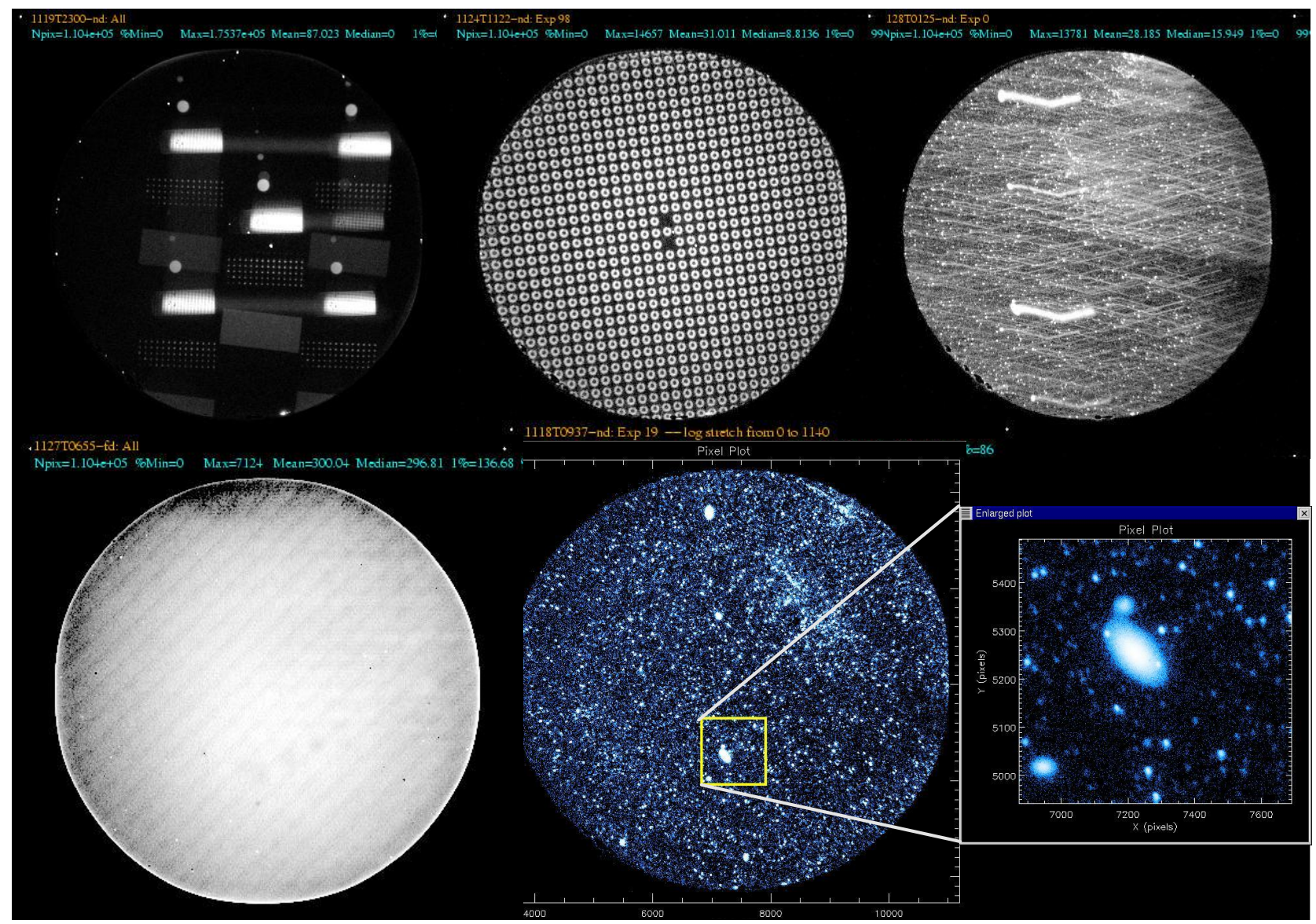

Figure 9 - Calibration images. From top left, clockwise: Fine grid array image in 5 positions. Defocused relative sensitivity test. Scan test of simulated sky target. Flat field exposure. Simulated sky target with blowup.

Among calibration parameters, we gave priority primarily to those that were more difficult to measure in flight. We adhered to the principle "test it like we fly it," to the extent practical. We commanded the instrument and downloaded data using the Orbital Sciences Corp. MAESTRO command and telemetry software that we will use in flight. The MAESTRO system communicated with a spacecraft simulator, which communicate with the instrument via a 1553 bus, and included a solid-state recorder simulator, which read science data through an RS-422 bus. We illuminated the instrument with a full aperture beam produced by a flight-spare telescope with a target wheel at its focus. A Roper Scientific Acton Research VM-502 0.2-m vacuum monochromator with a deuterium lamp provided UV illumination.

Our top priority calibration items were relative sensitivity versus wavelength, flat field, imaging-to-spectroscopic differential sensitivity versus wavelength, and spatial nonlinearity. The middle priority were absolute sensitivity (3 pencil beam locations in the aperture), grism dispersion function compared to imaging, high count rate tests (local and global), and a sky-simulation target. The lowest priority were PSF characterization, near-angle stray light, deuterium 
spectrum (monochromator at zero order), and detector background. At daily status/scheduling meetings, we adjusted the time allocation between tests based on their priority, on considerations of granularity and signal-to-noise, on the need to repeat certain tests at different environmental or detector temperatures, and on the relative efficiency of particular tests. Of the tests listed above, the local high count rate and near-angle stray light tests were reduced in scope because of operational difficulties. All other tests were performed satisfactorily. Much of the data has been analyzed and all critical parameters are adequately known and acceptable. Key performance results are summarized in Figure 8 and Table 2.

Table 2 - Instrument Design \& Performance Summary (based on Ground Calibration)

\begin{tabular}{|c|c|c|}
\hline Telescope Aperture & \multicolumn{2}{|c|}{$50 \mathrm{~cm}$} \\
\hline Optical Design & \multicolumn{2}{|c|}{$\begin{array}{l}\text { Modified Ritchey-Chrétien with } 4 \text { channels: FUV \& NUV Imaging, FUV \& } \\
\text { NUV Spectroscopy. FUV \& NUV obtained simultaneously using dichroic beam } \\
\text { splitter also acting as a field aberration corrector. }\end{array}$} \\
\hline Field of View & \multicolumn{2}{|c|}{1.2 degrees, circular } \\
\hline Focal Length & \multicolumn{2}{|c|}{$3 \mathrm{~m}$} \\
\hline Telescope coatings & \multicolumn{2}{|c|}{$\mathrm{Al}+\mathrm{MgF}_{2}$} \\
\hline Imaging/Grism Modes & \multicolumn{2}{|c|}{$\begin{array}{l}\text { Optics wheel with } \\
\text { (1) } \mathrm{CaF}_{2} \text { Imaging window, (2) } \mathrm{CaF}_{2} \text { transmission grism; (3) Opaque position. }\end{array}$} \\
\hline Grism Rotation & \multicolumn{2}{|c|}{$\begin{array}{c}\text { Grism position angle may be selected with a resolution of } 0.3 \text { degrees, } \\
\text { independent of } S / C \text { roll }\end{array}$} \\
\hline \multirow[t]{2}{*}{ Dichroic/Corrector } & \multicolumn{2}{|c|}{$\begin{array}{c}\text { Aspheric astigmatism corrector } \\
\text { Ion-etched fused silica (aspheric surfaces on both sides) } \\
\text { Dichroic beam splitter with dielectric multilayer coating on input side }\end{array}$} \\
\hline & FUV Channel & NUV Channel \\
\hline Band & $1350-1750 \AA$ & $1750-2800 \AA$ \\
\hline Beam path & Reflected from dichroic & Transmitted through dichroic \\
\hline Filters & $\begin{array}{c}\text { Blue edge filter } \\
\text { (blocks OI, Ly } \alpha \text { - transmission) }\end{array}$ & $\begin{array}{c}\text { Red block filter/Fold mirror } \\
\text { (blocks red Zodaical light) }\end{array}$ \\
\hline Detectors & \multicolumn{2}{|c|}{ Sealed tube Z-stack microchannel plate with crossed delay-line anodes } \\
\hline Detector Window & $\begin{array}{l}\qquad \mathrm{MgF}_{2} \\
\text { includes power for field flattering }\end{array}$ & $\begin{array}{c}\text { Fused Silica } \\
\text { includes power for field flattering }\end{array}$ \\
\hline Detector Photocathode & CsI, opaque, repeller grid on window & $\begin{array}{l}\mathrm{Cs}_{2} \mathrm{Te} \text {, semitransparent } \\
300 \mu \mathrm{m} \text { proximity gap }\end{array}$ \\
\hline Detector peak QE & $12 \%$ & $8 \%$ \\
\hline $\begin{array}{l}\text { Detector maximum local countrate w/o } \\
\text { temporary saturation within PSF }\end{array}$ & $\sim 100 \mathrm{c} / \mathrm{s}$ & $\sim 100 \mathrm{c} / \mathrm{s}$ \\
\hline System angular resolution & $\begin{array}{l}6.0 \operatorname{arcsec}(80 \% \text { EE diam) } \\
4.0 \operatorname{arcsec}(\text { FWHM })\end{array}$ & $\begin{array}{l}8.0 \operatorname{arcsec}(80 \% \text { EE diam) } \\
5.6 \operatorname{arcsec}(\text { FWHM })\end{array}$ \\
\hline Spectral Resolution & $250-300$ & $80-150$ \\
\hline Imaging Effective Area & $25 \mathrm{~cm}^{2}$ & $44 \mathrm{~cm}^{2}$ \\
\hline Spectroscopy Effective Area & $20 \mathrm{~cm}^{2}$ & $39 \mathrm{~cm}^{2}$ \\
\hline
\end{tabular}




\section{SPACECRAFT}

The GALEX spacecraft was developed by Orbital Sciences Corporation. It is based on a common bus architecture that was used on OrbView-4 (which did not achieve orbit) and will on the SORCE satellite (to launch winter 2001-2002). The largely single-string spacecraft consists of three major control blocks. The Power Regulation Electronics (PRE) controls power management and monitoring, and battery charging and discharging. The Central Electronics Unit (CEU) provides overall control of the spacecraft, command management, attitude control, and acts as the interface to uplink and downlink systems. The CEU central processor is a RAD-6000. The Attitude and Power Electronics (APE) furnishes safehold attitude control and a backup bus controller in the event of CEU faults. The APE, CEU, instrument DPU, and star-tracker share a common MIL-STD-1553 dual-string bus. High rate instrument science data is linked to the spacecraft's solid state recorder by a 16 bit RS-422 interface.

The power system includes a $3.1 \mathrm{~m}^{2}$ GaAs solar array with four panels fixed to the spacecraft, wrapped around the spacecraft at launch and with planned deployment during the first orbit. Eclipse power is provided by one 16 AH, 22 cell (11 common pressure vessels) $\mathrm{NiH}$ battery. The PRE provides power on/off switching under flight software or ground command, 6 solar array shunt switch units controlled by flight software, and sensors for battery, array, and load currents. APE software controls power load-shed during satellite safehold contingencies.

The RF system consists of a dual-string SIL S-band receiver and omni antenna for 2 kbps command uplink, an L3 Conic $\mathrm{X}$-band transmitter for 24 Mbps downlink, and a backup SIL S-band transmitter. All RF equipment is interfaced to the CEU uplink and downlink cards. The uplink card can operate autonomously with the APE in the event of CEU faults.

The attitude and control system (ACS) is three-axis stabilized. It consists of four Ithaco reaction wheel assemblies, eight course sun sensors, one three-axis magnetometer, three magnetic torquers (two bars and one coil), and a Ball CT-633 star tracker. Two inertial reference units are provided: one RGA20 Ring Laser Gyro, and one Litton Hemispherical Resonator SIRU upgraded from the Clark spacecraft unit. The primary attitude control software is loaded in the CEU, while safehold attititude control software is housed in the APE. The ACS provides course sun pointing on the day side, slewing to a science target during a 6 minute twilight scan, target absolute pointing accuracy of 5 arcminutes, relative pointing errors of 0.3 arcseconds (rss) over 10 second windows, a spiral dither at a few arsceconds/s during deep pointings, and a series of linked 23 degree scans during the all-sky survey. Following each eclipse, the ACS returns the satellite to solar-array sun pointing attitude.

\section{MISSION AND SCIENCE OPERATIONS AND DATA ANALYSIS}

Mission Operations will be performed at Orbital Science Corporation's Mission Operations Center (MOC) in Dulles, VA. Ground contacts will occur four times per day over the Universal Space Networks (USN) Perth and Hawaii stations. Satellite and instrument housekeeping will be monitored during ground contacts. Real time commanding will be used only during In-Orbit Checkout (IOC) and during infrequent critical command sequences-including anomaly recovery.

GALEX will be launched into low-earth orbit (690 km altitude, 29 deg inclination, 96 minute orbital period). Lowbackground requirements limit science observations to orbital night. During a typical orbit, the GALEX detectors are ramped to their nominal high voltage at night entry and ramped down to a safe low state prior to night exit. GALEX detectors are also ramped to safe levels during South Atlantic Anomaly passages, and during scans over bright stars during the All-sky Survey. Bright objects such as the sun, moon, earth limb and bright planets are avoided during observation intervals.

Science observations for the GALEX mission are planned at the Science Operations Center at Caltech in Pasadena, CA. Once per week, an observation description file containing a time-ordered sequence of pointing and instrument setting commands is sent to the Mission Operations Center. GALEX science mission planning software is designed to schedule the science surveys as efficiently as possible while ensuring that no operational or scientific observing constraints are violated. During each observation the GALEX instrument stores time-tagged photon positions for each detector on the spacecraft solid-state recorded. The $\sim 5 \mathrm{~GB}$ of science data collected each day will require four $25 \mathrm{Mbps} \mathrm{X}$-band downlink contacts to transmit to the ground for processing. 
The GALEX data analysis pipeline operated at the Science Operations Center receives the time-tagged photon lists, instrument/spacecraft housekeeping and satellite aspect information within two days of the ground contact. From these data sets, the pipeline reconstructs the aspect vs. time and generates images, spectra and source catalogs. The first pipeline module corrects the photon positions for detector and optical distortions and uses a maximum-entropy algorithm to calculate an optimal aspect solution based on the time-tagged photon data. A photometric module accumulates the photons into count and intensity maps and extracts sources from images. A spectroscopic module uses image source catalog inputs to extract spectra of these sources from the multiple slitless grism observations. Realistic processing simulations indicate that eight installed $1-2 \mathrm{GHz}$ dual processor servers running under the Linux operating system will process the complete set of data from a 24 hour period in considerably less than the 12 hour maximum requirement.

Final GALEX images and catalogs are to be released to the public at the Multimission Archive at Space Telescope (MAST) using a queriable database schema. The GALEX archive design is based on the Sloan Digital Sky Survey (SDSS) SkyServer architecture. This is a natural match, both because the GALEX science depends on SDSS corollary data and since the structure and the format of the data has remarkable similarities to the SDSS (multiwavelength, combination of imaging and spectroscopy, etc.). The Archive will be implemented using Microsoft SQL Server, and much of the front end will be web-based, probably implemented using SOAP and .NET, and adhering to the National Virtual Observatory standards.

\section{ACKNOWLEDGMENTS}

Numerous people have made important contributions to the GALEX program. A partial list of those include Tom Mitchie, Bill Price, Frank Street, Neil Dahya, and George Dorsey at Orbital Sciences Corporation, Mark Balzer, Ellyn McCoy, Bob Debusk, Larry Wild, Dave Rice, Brad Drake, Brad Swenson, Mike Phillips, Michelle Coleman, Don Moore, Larry Hovland, Patrick Noone, Richard Parks, Bill Harris, Jeff Oseas, John Wirth, Karen L'heureux, Mike Johnson, Terry Scharton, Krung Chang, Ted Iskenderian, Bob Hobson, Doug Packard, Kerry Erickson, Robin Dumas, Tien Nguyen, Tom Gavin, Larry Simmons, and Charles Elachi at JPL, Muamer Zukic at Cascade Optics, John Stone at Southwest Research Institute, Danielle Bonnet, Michel Laget, Jean-Michel Deharveng, Marie Treyer at Laboratorie Astrophysique Marseille, Bud Hill at Baja Research, Mark Gummin, Alias Aerospace, Darrel Doliber, Sharon Jelinsky, Jim Malloy, Bojan Turko, Rob Abiad, and Daniel Blackman at U.C. Berkeley, Minerva Calderon, John Klemic, Mark Maechtlen, Hilary Caisley, Janester Short, Monica Torres, Jaewoo Lee, Suk-yong Yi, Diane Engler, Tom Tombrello, and Steve Kaye at Caltech, Jeffrey Gumm, Bill Davis, Frank Snow, Jim Barrowman, Tony Cambriote at GSFC, and Philippe Crane, Hashima Hasan, George Albright, Anne Kinney, and Ed Weiler at NASA Headquarters.

\section{REFERENCES}

1. C. Martin, et al, "The Galaxy Evolution Explorer", in Ultraviolet-Optical Space Astronomy Beyond HST, ASP Conf. Series Vol. 164, ed. Morse, J.A., Shull, J.M, and Kinney, A.L.

2. I.S. Bowen and A.H. Vaughan, 1973, PASP, 85, 104.

3. R. Grange et al. 2001, ICS0 2000 proceedings, 4th International Conference On Space Optics, CNES-Mediatec Diffusion, p.91.

4. Mercier et al. 2001, ICS0 2000 proceedings, 4th International Conference On Space Optics, CNES-Mediatec Diffusion, p.99.

5. P. Jelinsky, et al., "The Galaxy Evolution Explorer Detectors", SPIE Conf. 4854, Future EUV-UV and Visible Space Astrophysics Missions and Instrumentation. 